\title{
Microsurgical rhizotomy for trigeminal neuralgia in MS patients: technique, patient satisfaction, and clinical outcomes
}

\author{
Mark G. Bigder, MD, MPH, ${ }^{1,2}$ Sandeep Krishnan, BSc, ${ }^{1}$ E. Francis Cook, ScD, ${ }^{2}$ and \\ Anthony M. Kaufmann, MD, MSc, FRCSC ${ }^{1}$ \\ ${ }^{1}$ Section of Neurosurgery, Department of Surgery, University of Manitoba, Winnipeg, Manitoba, Canada; and 2Department of \\ Epidemiology, Harvard T. H. Chan School of Public Health, Boston, Massachusetts
}

\begin{abstract}
OBJECTIVE Patients with multiple sclerosis (MS)-associated trigeminal neuralgia (TN) have higher recurrence and retreatment rates than non-MS patients. The optimal management strategy and role for microsurgical rhizotomy (MSR) for MS-TN remains to be determined. The aim of this study was to report time to treatment failure (TTF) and pain scores following MSR compared to percutaneous and Gamma Knife procedures.

METHODS Time to treatment failure was analyzed after MSR $(n=14)$ versus prior procedures $(n=53)$ among MS-TN patients. Kaplan-Meier curves and log-rank test were utilized to compare TTF after MSR versus prior procedures using the same cohort of patients as their own control group. Subsequent analysis compared TTF after MSR to TTF after 93 other procedures among a second cohort of $18 \mathrm{MS}-\mathrm{TN}$ patients not undergoing MSR. BNI pain scores were compared between MSR and other procedures among the MS-TN cohort using a chi-square test.

RESULTS TTF was significantly longer after MSR than after other procedures in the MSR cohort (median TTF 79 vs 10 months, respectively, $p<0.0001)$. Similarly, TTF was longer after MSR than after prior procedures in the non-MSR cohort (median TTF 79 vs 13 months, respectively, $p<0.001$ ). MSR resulted in a higher proportion of excellent pain scores when compared to other procedures in the non-MSR cohort (77\% vs $29 \%, p<0.001)$. Probability of treatment survival was higher after MSR than after other procedures at all time points $(3,6,12,24,36$, and 48 months). There were no deaths or major complications after MSR.
\end{abstract}

CONCLUSIONS TTF was significantly longer following MSR compared to prior procedures in MS-TN patients. Additionally, a higher proportion of patients achieved excellent BNI pain scores after MSR.

https://thejns.org/doi/abs/10.3171/2017.12.JNS171647

KEYWORDS trigeminal neuralgia; multiple sclerosis; partial sensory rhizotomy; refractory trigeminal neuralgia; microsurgical rhizotomy; Dandy procedure; pain

$\mathrm{T}$ RIGEMINAL neuralgia (TN) is characterized by severe, unilateral, paroxysmal pain attacks in the distribution of one or more divisions of the trigeminal nerve. ${ }^{11} \mathrm{TN}$ has an annual incidence range of 4-5 per 100,000 persons per year ${ }^{15}$ and prevalence of $3-5$ per 100,000; the prevalence is higher in the population of patients with multiple sclerosis (MS), occurring in approximately $3 \%-7 \%$ of MS patients. ${ }^{2,9}$ Therapeutic options for TN include medical therapy, percutaneous balloon compression, glycerol rhizotomy and radiofrequency ablation, stereotactic radiosurgery, microvascular decom- pression (MVD), and Walter Dandy's technique of surgical rhizotomy. In the evolution of neurosurgical practice, Dandy's early technique of partial sensory rhizotomy of the trigeminal nerve has fallen out of favor for the treatment of most cases of classic TN. Surgical rhizotomy has been largely replaced by MVD as well as percutaneous techniques for the management of most cases of TN. In a subgroup of TN patients, such as those with MS-associated TN (MS-TN), treatment can be particularly challenging, as these patients reportedly have earlier onset of symptoms and a higher likelihood of bilateral involvement

ABBREVIATIONS BNI = Barrow Neurological Institute; GKS = Gamma Knife surgery; MS = multiple sclerosis; MSR = microsurgical rhizotomy; MS-TN = MS-associated $\mathrm{TN} ; \mathrm{MVD}=$ microvascular decompression; TN = trigeminal neuralgia; TREZ = trigeminal root entry zone; TTF = time to treatment failure.

SUBMITTED July 10, 2017. ACCEPTED December 19, 2017.

INCLUDE WHEN CITING Published online July 13, 2018; DOI: 10.3171/2017.12.JNS171647. 


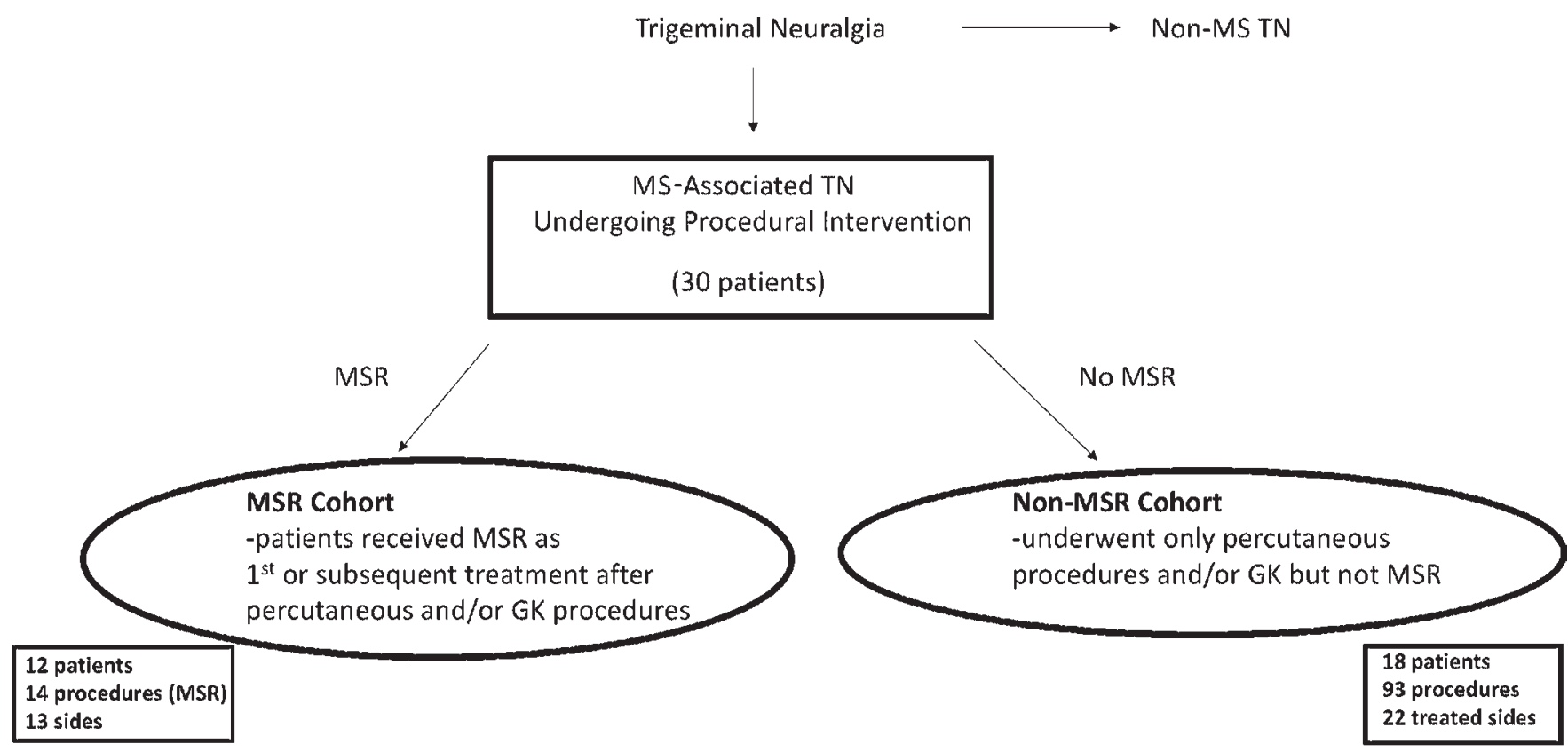

FIG. 1. Study populations of interest. Patients who underwent MSR by the senior author made up the MSR cohort. These patients may have received MSR as a first procedure or following previous percutaneous and/or Gamma Knife (GK) procedures. The results of MSR in this cohort were initially compared to the results of prior procedures in the MSR cohort and subsequently to the results of prior procedures in the non-MSR cohort, which comprised all patients treated in Manitoba for MS-TN with various procedures other than MSR.

as well as higher rates of treatment failure, recurrence, and repeat procedures and shorter pain-free intervals between treatments. ${ }^{710,12,14,21}$ Microvascular decompression is not an option for most MS patients, as the pathophysiology of pain in this patient group is typically not related to neurovascular compression and thus most often not amenable to decompression as in patients with classic TN. Thus, MS patients afflicted with TN commonly endure years of minimally effective pain relief from medications and undergo multiple procedural interventions. For this select group of patients, microsurgical rhizotomy (MSR), often referred to as partial sensory rhizotomy or Dandy procedure, may offer more effective, durable, and cost-effective pain relief than medical management or other surgical procedures. Recent literature regarding the safety, efficacy, and durability of microsurgical partial sensory rhizotomy is sparse, ${ }^{1,3}$ particularly in the MS population, and thus optimal management of this patient group remains unclear. We describe our surgical outcomes of MSR in a consecutive series of MS-TN patients who underwent partial sensory rhizotomy performed by the senior author (A.M.K.).

\section{Methods}

\section{Patient Population}

This study is a retrospective review of all consecutive patients (14) who underwent neurosurgical intervention for MS-associated TN (MS-TN) between 1997 and March 2017 performed by the senior author (A.M.K.). Among this group of patients, those undergoing MSR (open surgery) for MS-TN constituted the first group. Patients were selected for surgery on the basis of previous multimodal- ity treatment failure and/or persistent severe neuralgia defined as Barrow Neurological Institute (BNI) pain score of 4 or 5 , or medication intolerance in the context of persistent pain. The majority of surgeries were performed at the Centre for Cranial Nerve Disorders at the Health Sciences Centre in Winnipeg, Manitoba, Canada. One surgery prior to November 2000 was performed at Foothills Medical Centre in Calgary, Alberta, by the senior author (A.M.K.). Clinical results after MSR in this group of patients were compared to results in a second cohort of MS-TN patients (18) who were treated consecutively by the senior author with various other procedures (nonopen) but did not undergo MSR (Fig. 1).

\section{Data Collection}

All MSR procedures performed by the senior author were recorded in a prospective database along with surgical diagrams. Clinic and hospital records were reviewed for the purpose of determining clinical outcome measures, including extent and duration of pain relief, ongoing requirement for pain medication, subsequent procedures, and complications. A patient satisfaction survey was designed and administered to patients in an effort to gauge patient satisfaction with surgery as well as to attain clinical follow-up data. Most recent follow-up data were obtained via telephone interview and patient questionnaire. Pain outcome and sensory deficit were assessed based on the BNI pain intensity score and BNI Facial Numbness Scale, respectively.

\section{Surgical Technique}

We utilize our standard retrosigmoid MVD surgi- 
cal technique to gain access to the trigeminal root entry zone (TREZ). ${ }^{4,6,8,13}$ Intraoperative monitoring is utilized throughout the procedure, including brainstem auditory evoked potentials. Exploration of the TREZ is carried out to identify any potential neurovascular conflict. In cases of questionable neurovascular compression, the culprit vessel is mobilized away from the nerve and held in place with a Teflon implant. Approximately $2 \mathrm{~mm}$ distal to the trigeminal root entry to the pons, the caudal one-half or one-third of the trigeminal nerve fibers is divided along the length of the nerve for cases involving V2/3 or V3, respectively. In cases of V1 pain, the rostral one-third of the nerve is selectively sectioned. We utilize a no. 3 Jannetta dissector, often in combination with a no. 8 Jannetta, for the separation and dissection of the nerve followed by a beaver blade or microscissors to section the nerve (Fig. 2 and Video 1).

VIDEO 1. Video clip showing an intraoperative microscopic view in a patient with V3 distribution pain undergoing separation of the caudal third of the trigeminal nerve with a no. 3 Jannetta dissector followed by partial sensory rhizotomy of the separated fascicles with microscissors. Copyright Mark Bigder. Published with permission. Click here to view.

\section{Clinical Outcome Measure}

The primary outcome of interest was time to treatment failure (TTF), defined as the time interval between the index surgical procedure and a repeat procedure if required. The indication for retreatment was recurrence of pain with BNI 4 or 5 severity or medication intolerance in the context of persistent pain. Retreatment is typically offered within 1-2 weeks in cases of severe pain recurrence. Secondary outcome measures included complications such as wound infection, meningitis, cerebrospinal fluid leak, keratitis, and anesthesia dolorosa; clinical outcomes such as BNI pain and sensory scores; time to pain recurrence; and results of the patient satisfaction survey. BNI pain scores of 1 and 2 were considered to indicate excellent outcomes, scores of $3 \mathrm{a}$ and $3 \mathrm{~b}$ were considered to indicate good outcomes, and BNI scores of 4 and 5 were considered to indicate poor outcomes. Complications and clinical outcomes were assessed through review of hospital charts as well as through clinical and telephone follow-up visits and the patient satisfaction survey, which was administered by telephone by a neurosurgery nurse not involved in the study.

\section{Data Analysis}

Statistical analysis was performed utilizing Stata statistical software (release 14, StataCorp LP). Kaplan-Meier survival curves were utilized to graph TTF in the study cohort of MS patients undergoing MSR versus TTF for all previous procedures in the same patients. We subsequently analyzed MSR versus Gamma Knife stereotactic radiosurgery (Gamma Knife surgery [GKS]) as the first procedure performed in this study group and also compared MSR versus percutaneous rhizotomy as the first treatment. Logrank tests were utilized to compare curves. Subsequently, TTF and BNI pain scores were compared after MSR to those from all procedures performed in a second group of MS-TN patients who were managed at our institution but did not undergo MSR (non-MSR group). A two-sided Student t-test and chi-square test were utilized where ap-

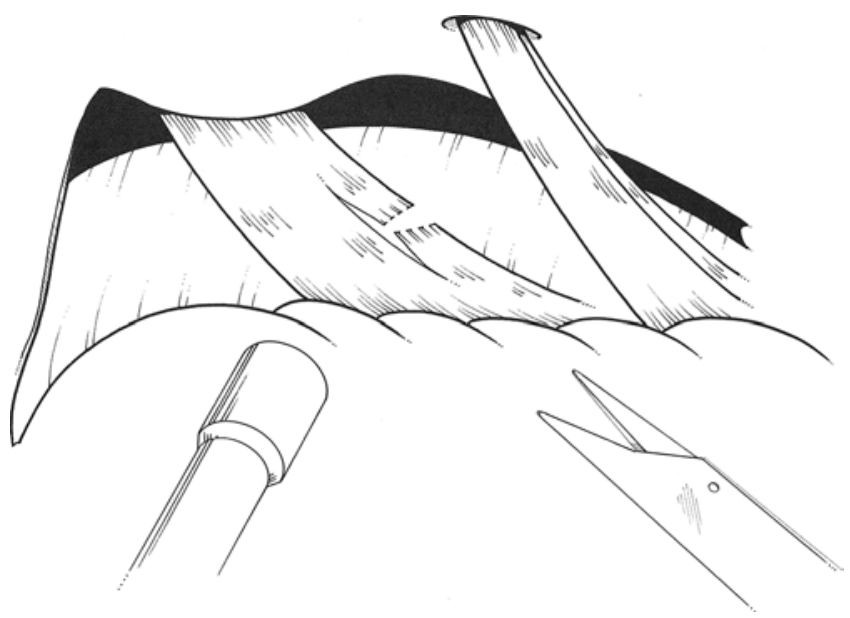

FIG. 2. Schematic diagram of an intraoperative microscopic view in a patient with right $\mathrm{V} 3$ distribution pain undergoing MSR of the caudal third of the trigeminal nerve. The nerve fascicles have been separated utilizing Jannetta no. 3 and no. 8 dissectors. Microscissors are utilized at this point to perform the rhizotomy. Copyright Jon Stepaniuk. Published with permission.

propriate to compare patient baseline characteristics between the two cohorts as well as differences in BNI pain scores. A cutoff $p$ value of $<0.05$ was considered to be statistically significant.

\section{Results \\ Patient Characteristics}

There were 14 MSR procedures performed on 13 sides in 12 patients ( 3 men and 9 women), who constituted the MSR cohort (Table 1); 1 patient had 2 sides treated, and 1 patient underwent 2 procedures on the same side. The patients' mean age was 59.4 years (range $48-76$ years) at the time of MSR (Table 2). The mean number of procedures prior to MSR was 4.5 (median 4, range 0-10). Thirteen percutaneous glycerol rhizotomy procedures were performed in 5 patients, 24 percutaneous balloon compression rhizotomies in 7 patients, 7 radiofrequency rhizotomies in 4 patients, and 9 Gamma Knife procedures in 6 patients. The mean duration of TN symptoms prior to MSR was 7.8 years (median 5.9 years, range $0.8-18.2$ years). All patients had a BNI pain intensity score of 5 prior to MSR.

Among the patients who did not undergo MSR (nonMSR cohort), there were 8 male and 10 female patients, for a total group size of 18 patients who underwent various percutaneous rhizotomy procedures and GKS but did not undergo MSR. There were 93 procedures performed on 22 sides in this group. The patients' mean age at the time of last procedure was 61.3 years (range 46-78 years) with a mean duration of $\mathrm{TN}$ prior to the last procedure of 7.6 years (median 6.9 years, range $2.5-19.9$ years). The mean number of procedures prior to last treatment was 2.1 (median 2, range $0-10$ ).

When the patients in the MSR cohort were compared to those in the non-MSR cohort for differences in baseline characteristics, there was no significant difference in sex, age, or duration of TN between the 2 groups (Table 3 ). The 
TABLE 1. Summary of demographic and clinical characteristics of the MSR cohort

\begin{tabular}{|c|c|c|c|c|c|}
\hline ID & $\begin{array}{l}\text { Pt Age } \\
\text { (yrs), Sex }\end{array}$ & $\begin{array}{l}\text { Duration of } \\
\text { TN (mos) }\end{array}$ & $\begin{array}{l}\text { Total No. \& Type of Prior } \\
\text { Procedures* }\end{array}$ & $\begin{array}{l}\text { Complications From Prior } \\
\text { Procedures }\end{array}$ & Complications From MSR \\
\hline $1 \mathrm{~L}$ & $52, \mathrm{~F}$ & 10 & 10: PGR (3), PBCR (6), GKS (1) & & Mild, sporadic dysesthesia \\
\hline $1 \mathrm{R}$ & $56, F$ & 14 & 5: PBCR (2), GKS (2), MVD (1) & & \\
\hline 2 & $48, M$ & 65 & 5: RFR (1), PBCR (3), GKS (1) & Corneal abrasion \& tarsorrhaphy & \\
\hline 3 & $62, \mathrm{~F}$ & 93 & 3: RFR (3) & Corneal abrasion \& tarsorrhaphy & \\
\hline 4 & $76, \mathrm{~F}$ & 49 & None & & \\
\hline 5 & $49, M$ & 12 & None & & Wound infection \\
\hline 6 & $66, \mathrm{~F}$ & 200 & 4: PBCR (2), PGR (1), GKS (1) & & \\
\hline 7 & $66, M$ & 58 & 3: PBCR (2), GKS (1) & & \\
\hline 8 & $49, \mathrm{~F}$ & 204 & 8: PGR (3), PBCR (3), GKS (2) & & \\
\hline 9 & $60, \mathrm{~F}$ & 218 & 3: local anesthetic injections & & \\
\hline 10 & $65, \mathrm{~F}$ & 119 & 9: PGR (5), RFR (1), PBCR (3) & & $\begin{array}{l}\text { Moderately bothersome } \\
\text { dysesthesia }\end{array}$ \\
\hline 11 & $68, \mathrm{~F}$ & 71 & 5: RFR (2), PGR (1), GKS (1) & & Mild, sporadic dysesthesia \\
\hline 12 & $55, \mathrm{~F}$ & 107 & 3: PBCR (3) & & \\
\hline
\end{tabular}

GKS = Gamma Knife surgery; $\mathrm{L}=$ left; $\mathrm{MVD}$ = microvascular decompression; $\mathrm{PBCR}$ = percutaneous balloon compression rhizotomy; PGR = percutaneous glycerol rhizotomy; $P t=$ patient; $R=$ right; RFR = radiofrequency rhizotomy.

Data are reported for 12 patients who underwent a total of 14 MSR procedures. One patient underwent MSR on the left and right sides, represented here as $1 \mathrm{~L}$ and $1 R$, and 1 patient (patient 11) underwent 2 MSR procedures on the same side. The reported age is the age of the patient at the time of MSR (initial MSR for patient 11).

*Values in parentheses indicate number of prior procedures of the specified type.

MSR cohort, however, had a significantly higher number of prior procedures, with a mean of 4.5 compared to 2.1 in the non-MSR cohort $(\mathrm{p}=0.02)$.

\section{Follow-Up}

The mean duration of follow-up after MSR was 36.5 months (median 28.5 months, range 1-130 months). Patient 1 passed away from an unrelated cause 4 months following MSR for her second involved side; this patient was censored at 1 month in the survival analysis and postoperative BNI scores at this time point were utilized for analysis in short-term outcomes. Subsequent review of records revealed documentation that the patient maintained a BNI pain score of 1 at 4 months postoperatively, prior to her death. There were 3 additional patients with less than 1 year of follow-up: patients 9, 10, and 11 (for her second MSR procedure). Patients 9 and 10 underwent MSR at 4 and 6 months, respectively, prior to the writing of this paper with updated follow-up; patient 9 was pain free during the early postoperative and last follow-up and was on a substantially reduced dose of preoperative medications and in the process of tapering, whereas patient 10 was pain free and completely off preoperative medications. Patient 11 underwent a second MSR for recurrent pain 22 months after the initial MSR procedure; this patient was initially pain free and off medications after the first surgery. The 9-month follow-up after the second MSR occurred at the time of writing, and at that time the patient was pain free and off medications.

\section{Short-Term Postoperative Outcomes}

All patients undergoing MSR were evaluated in the immediate postoperative period (1-2 days) and followed up by telephone or clinic visit at 1-2 weeks and 6 weeks postoperatively. Postoperative clinical outcomes and operative details for the patients in the MSR cohort are summarized in Table 4. Comparison of postprocedure BNI pain scores was made between MSR patients and patients treated with other procedures among the non-MSR cohort (Table 5). All MSR patients had symptom improvement at short-term follow-up and had BNI pain scores of 1-3a. A BNI pain score of 1 was achieved after 12/14 (85.7\%) performed procedures; after the other 2 procedures the patients had BNI pain scores of $3 \mathrm{a}$ and were on substantially reduced, tapering doses of gabapentin.

\section{Surgical Complications}

No patients experienced any major operative complications or adverse neurological changes after MSR. One patient developed a wound infection in the days following surgery and was treated with a short course of antibiotics without further complication. No patient experienced appreciable trigeminal motor loss.

\section{Sensory Changes}

BNI sensory scores of 1 or 2 were observed after 13/14 MSR procedures (score of 1 in 2 cases and score of 2 in 11 cases). One patient experienced what was vaguely described as "somewhat bothersome" facial numbness after surgery, with a BNI sensory score of 3; this patient had the caudal half of the trigeminal nerve root sectioned. In comparison to preexisting neuralgia symptoms, however, the patient clarified that this numbness was perceived to be negligible and not associated with any pain or drooling. The majority of patients (11/12) did not report any bothersome facial numbness after MSR. 
TABLE 2. Summary of baseline characteristics and duration of post-MSR follow-up for the MSR cohort

\begin{tabular}{|c|c|}
\hline Characteristic & Value \\
\hline \multicolumn{2}{|l|}{ Sex } \\
\hline Male & $3 / 12(25 \%)$ \\
\hline Female* $^{*}$ & $9 / 12(75 \%)$ \\
\hline \multicolumn{2}{|l|}{ Age (yrs) } \\
\hline Mean & 59.4 \\
\hline Range & $48-76$ \\
\hline \multicolumn{2}{|c|}{ No. of prior procedures (per side) } \\
\hline Mean & 4.5 \\
\hline Median & 4 \\
\hline Range & $0-10$ \\
\hline \multicolumn{2}{|c|}{ Mean duration of TN prior to op (mos) } \\
\hline Mean & 93.8 \\
\hline Median & 71 \\
\hline Range & $10-218$ \\
\hline \multicolumn{2}{|l|}{ FU time after MSR (mos) } \\
\hline Mean & 36.6 \\
\hline Median & 28.5 \\
\hline Range & $1-130$ \\
\hline \multicolumn{2}{|c|}{ Preop pain distribution (sides) } \\
\hline V1 & $0 / 13$ \\
\hline V2 & $0 / 13$ \\
\hline V3 & $5 / 13$ \\
\hline $\mathrm{V} 1+\mathrm{V} 2$ & $1 / 13$ \\
\hline $\mathrm{V} 2+\mathrm{V} 3$ & $6 / 13$ \\
\hline $\mathrm{V} 1+\mathrm{V} 2+\mathrm{V} 3$ & $1 / 13$ \\
\hline
\end{tabular}

$\mathrm{FU}=$ follow-up.

* One female patient underwent MSR on both sides (at different time points), as shown in Table 1. Thus, analyzed by sides treated, patient sex was male for 3 sides and female for 10 sides.

\section{Deafferentation Pain}

No patient experienced anesthesia dolorosa or severe deafferentation pain following MSR. Three patients reported occasional intermittent discomfort in the region of previous neuralgia distribution but affirmed that these sensations did not resemble the previous neuralgia pain they had experienced prior to surgery and in comparison were negligible. Two patients described these sensations as a mild tingling at times, while 1 described them as "moderately bothersome." Two of these patients did not find the sensations themselves necessarily bothersome but reported that they elicited feelings of "anxiety" and "fear" that their neuralgia might return.

\section{Long-Term Outcomes}

The horizontal line graph in Fig. 3 demonstrates time to treatment failure (TTF) and procedure type for individual patients. Kaplan-Meier curves were utilized to analyze TTF, defined as the time interval between a procedure and the need for a subsequent procedure. TTF was compared for the MSR procedure versus TTF after any other type of procedure, including GKS, percutaneous glycerol,
TABLE 3. Comparison of patient baseline characteristics in the MSR and non-MSR cohorts

\begin{tabular}{llll}
\hline \multicolumn{1}{c}{ Characteristic } & $\begin{array}{c}\text { MSR } \\
\text { Cohort }\end{array}$ & $\begin{array}{c}\text { Non-MSR } \\
\text { Cohort }\end{array}$ & $\begin{array}{c}\mathrm{p} \\
\text { Value }\end{array}$ \\
\hline Sex & & & \\
\hline Male & $3 / 12(25 \%)$ & $8 / 18(44 \%)$ & 0.28 \\
\hline Female & $9 / 12(75 \%)$ & $10 / 18(56 \%)$ & 0.28 \\
\hline Age (yrs) & & & 0.73 \\
\hline Mean & 59.4 & 61.3 & \\
\hline Range & $48-76$ & $46-78$ & \\
\hline No. of prior procedures (per side) & & & 0.02 \\
\hline$\quad$ Mean & 4.5 & 2.1 & \\
\hline Median & 4 & 2 & \\
\hline Range & $0-10$ & $0-10$ & \\
\hline Mean duration of TN prior to op & & & \\
$\quad$ (mos) & & & \\
\hline$\quad$ Mean & 93.8 & 91.0 & \\
\hline$\quad$ Median & 71 & 83 & \\
\hline$\quad$ Range & $10-218$ & $30-239$ & \\
\hline
\end{tabular}

radiofrequency rhizotomy, and balloon compression rhizotomy, in the same patient group (MSR cohort) (Fig. 4). The log-rank test was utilized to compare the 2 curves, which showed that MSR had a significantly longer TTF than did prior procedures $(\mathrm{p}<0.0001)$. The median treatment survival after MSR was 79 months versus 10 months after either percutaneous rhizotomy or GKS, where treatment survival refers to persistent benefit from treatment and thus lack of necessity for retreatment. The probability of treatment survival was $100 \%$ at 1 year, $89 \%(95 \%$ CI $43 \%-98 \%)$ at 2 years, $89 \%(95 \%$ CI $43 \%-98 \%)$ at 3 years, and $74 \%$ (95\% CI 29\%-93\%) at 4 years after MSR and $38 \%$ (95\% CI 25\%-50\%), $11 \%$ (95\% CI 5\%-21\%), $4 \%$ (95\% CI 1\%-11\%), and 2\% (95\% CI 0\%-9\%) at 1,2, 3, and 4 years, respectively, after percutaneous rhizotomy or GKS.

In subgroup analysis of GKS versus MSR there was a statistically significant difference, with MSR having a longer TTF $(p=0.0007)$. The median treatment survival after MSR was 79 months compared to 16 months after GKS (Fig. 5).

There were 3 patients who underwent MSR as a first procedure. An analysis of TTF was performed for these 3 MSR procedures versus TTF after first treatment for the remaining 10 patient sides (all 10 were percutaneous procedures). Kaplan-Meier curves are demonstrated in Fig. 6. TTF was significantly longer after MSR than after percutaneous procedures $(\mathrm{p}=0.0196, \log$-rank test $)$.

In 3 of 13 initially treated sides (23.1\%), the patients eventually had recurrence of $\mathrm{TN}$ to preoperative pain intensity (BNI pain score 5)-79, 42, and 22 months postoperatively. All 3 of these patients required additional treatment, which is further discussed below. Another 3 patients experienced recurrence of some pain, which was adequately controlled with medication (BNI pain score 3b) at 46, 6, and 11 months after MSR.

Subsequently, a second analysis was performed in 
TABLE 4. Operative findings and clinical outcome in the MSR cohort

\begin{tabular}{|c|c|c|c|c|c|c|c|c|}
\hline ID & $\begin{array}{c}\text { Extent of } \\
\text { Rhizotomy }\end{array}$ & $\begin{array}{l}\text { Preop BNI-P \& } \\
\text { Distribution }\end{array}$ & $\begin{array}{l}\text { Postop } \\
\text { BNI-P }\end{array}$ & $\begin{array}{l}\text { BNI-P at } \\
\text { Last FU }\end{array}$ & $\begin{array}{l}\text { BNI-S at } \\
\text { Last FU }\end{array}$ & $\begin{array}{c}\text { Time to } \\
\text { Recurrence }\end{array}$ & $\begin{array}{c}\text { Time to } \\
\text { Retreatment }\end{array}$ & $\begin{array}{c}\text { Total FU } \\
\text { (mos) }\end{array}$ \\
\hline $1 \mathrm{R}$ & Caudal $50 \%$ & $5(\mathrm{~V} 3)$ & $1 \dagger$ & $1 \dagger$ & $2(\mathrm{~V} 2 / 3)$ & NA & NA & 53 \\
\hline $1 \mathrm{~L}$ & Caudal $50 \%$ & 5 (V3) & $1 \dagger$ & $1 \dagger$ & $2(\mathrm{~V} 2 / 3)$ & NA & NA & 1 \\
\hline 2 & Caudal 66\% & $5(\mathrm{~V} 2 / 3)$ & $1 \ddagger$ & $1 \ddagger$ & $2(\mathrm{~V} 2 / 3)$ & NA & NA & 25 \\
\hline 3 & Caudal 50\% & $5(\mathrm{~V} 3)$ & 1 & $3 b$ & 3 & 46 & NA & 46 \\
\hline 4 & Caudal $50 \%$ & 5 (V3) & 1 & 5 & 2 & 79 & 79 & 80 \\
\hline 5 & Caudal $40 \%$ & $5(\mathrm{~V} 3)$ & 1 & 5 & 2 (V3) & 42 & 42 & 42 \\
\hline 6 & Caudal $85 \%$ & $5(\mathrm{~V} 1 / 2 / 3)$ & 1 & $3 a$ & $2(\mathrm{~V} 1 / 2 / 3)$ & NA & NA & 32 \\
\hline 7 & Caudal $50 \%$ & $5(\mathrm{~V} 2 / 3)$ & 1 & $3 b$ & 2 & 6 & NA & 55 \\
\hline 8 & Caudal 66\% & $5(\mathrm{~V} 2 / 3)$ & $3 a \S$ & $3 a$ & 2 & NA & NA & 130 \\
\hline 9 & Caudal $50 \%$ & $5(\mathrm{~V} 2 / 3)$ & 3aף & $3 a$ & $2(\mathrm{~V} 2 / 3)$ & NA & NA & 2 \\
\hline 10 & Caudal $50 \%$ & $5(\mathrm{~V} 2 / 3)$ & 1 & 1 & $2(\mathrm{~V} 2 / 3)$ & NA & NA & 4 \\
\hline $11^{* *}$ & Caudal $50 \%$ & $5(\mathrm{~V} 2 / 3)$ & 1 & 5 & $1(\mathrm{~V} 1 / 2)$ & 20 & 22 & 22 \\
\hline $11 \dagger \dagger$ & Caudal $90 \%$ & $5(\mathrm{~V} 2 / 3)$ & 1 & 1 & $1(\mathrm{~V} 1 / 2)$ & NA & NA & 7 \\
\hline 12 & Rostral 80\% & $5(\mathrm{~V} 1 / 2)$ & 1 & $3 b$ & 2 & NA & NA & 13 \\
\hline \multicolumn{9}{|c|}{$\begin{array}{l}\text { BNI-P = BNI pain score; BNI-S = BNI sensory score; NA = not applicable. } \\
\text { * Portion of nerve sectioned. } \\
\text { † Patient did not require medication for TN, but continued to be treated with a reduced dose of oxcarbazepine and carbamazepine for a seizure } \\
\text { disorder. } \\
\text { † Patient required a reduced-dose carbamazepine regimen for contralateral TN pain. } \\
\text { § Pain free on reduced dose of gabapentin. } \\
\text { † Pain free on tapering, reduced dose of gabapentin. } \\
\text { ** First MSR procedure. Follow-up data in this row refer to follow-up after the first procedure and before the second. } \\
\text { †† Second MSR procedure. Follow-up data in this row refer to follow-up after the second procedure only. }\end{array}$} \\
\hline
\end{tabular}

which TTF for patients undergoing MSR was compared to TTF for all prior procedures in a second cohort of MS-TN patients who did not undergo MSR (Fig. 7). The median TTF was 79 versus 13 months in favor of MSR.

\section{Subsequent Surgical Procedures}

As mentioned above, 3 patients (patients 4, 5, and 11) required subsequent procedures after recurrence of pain to preoperative intensity levels (BNI pain score 5) at 79, 42 , and 22 months, respectively (mean 47.7 months). Patients 4 and 5 were retreated at institutions within their

TABLE 5. Postoperative BNI pain scores

\begin{tabular}{clcr}
\hline \multicolumn{1}{c}{ Score } & MSR Cohort & Non-MSR Cohort & p Value \\
\hline BNI 1-2 (no meds) & $10 / 13(77 \%)$ & $26 / 89(29 \%)$ & $<0.001$ \\
\hline BNI 1-3a & $13 / 13(100 \%)$ & $67 / 89(75.3 \%)$ & 0.043 \\
\hline BNI 1-3b & $13 / 13(100 \%)$ & $84 / 89(94.4 \%)$ & 0.381 \\
\hline BNI 1 & $10 / 13(77 \%)$ & $22 / 89(24.7 \%)$ & $<0.001$ \\
\hline BNI 2 & $0 / 13$ & $4 / 89(4.5 \%)$ & 0.436 \\
\hline BNI 3 & $3 / 13(23 \%)$ & $58 / 89(65.2 \%)$ & 0.004 \\
\hline BNI 3a & $3 / 13$ & $41 / 89(46.1 \%)$ & \\
\hline BNI 3b & NA & $17 / 92(19.1 \%)$ & \\
\hline BNI 4 or 5 & $0 / 13$ & $5 / 89(5.6 \%)$ & 0.381 \\
\hline BNI 4 & & $1(1.1 \%)$ & \\
\hline BNI 5 & $4(4.5 \%)$ & \\
\hline
\end{tabular}

home province as described below. At 79 months after MSR, patient 4 underwent percutaneous balloon compression rhizotomy followed by glycerol rhizotomy and GKS within the span of 2 weeks with no improvement in symptoms and a BNI pain score of 5 at last followup, which occurred shortly before death the same month due to an unrelated cause. She had been symptom and medication free (BNI pain score 1) for 79 months prior to recurrence. Patient 5 was pain free and off medications for 42 months (BNI pain score 1) prior to developing recurrence of symptoms and underwent 2 percutaneous balloon compression rhizotomies at 42 months with no symptom relief. This patient was lost to further follow-up at our center.

Patient 11 was pain and medication free for 11 months after initial treatment with MSR, at which point she developed recurrence of BNI pain score 5 pain. She underwent repeat MSR at our center with an excellent postoperative outcome and a BNI pain score of 1 at 7 months' follow-up.

\section{Patient Satisfaction Outcomes}

A patient satisfaction survey was administered by a neurosurgery clinical nurse with the aim of obtaining qualitative data to gauge clinical outcomes. Complete survey results were available for 6 of 12 patients, as this survey was administered in the months leading up to the writing of this paper and recent follow-up was not possible for the remaining patients. All 6 of the patients for whom data were available responded that they experienced a reduction in pain following surgery, would recommend the 


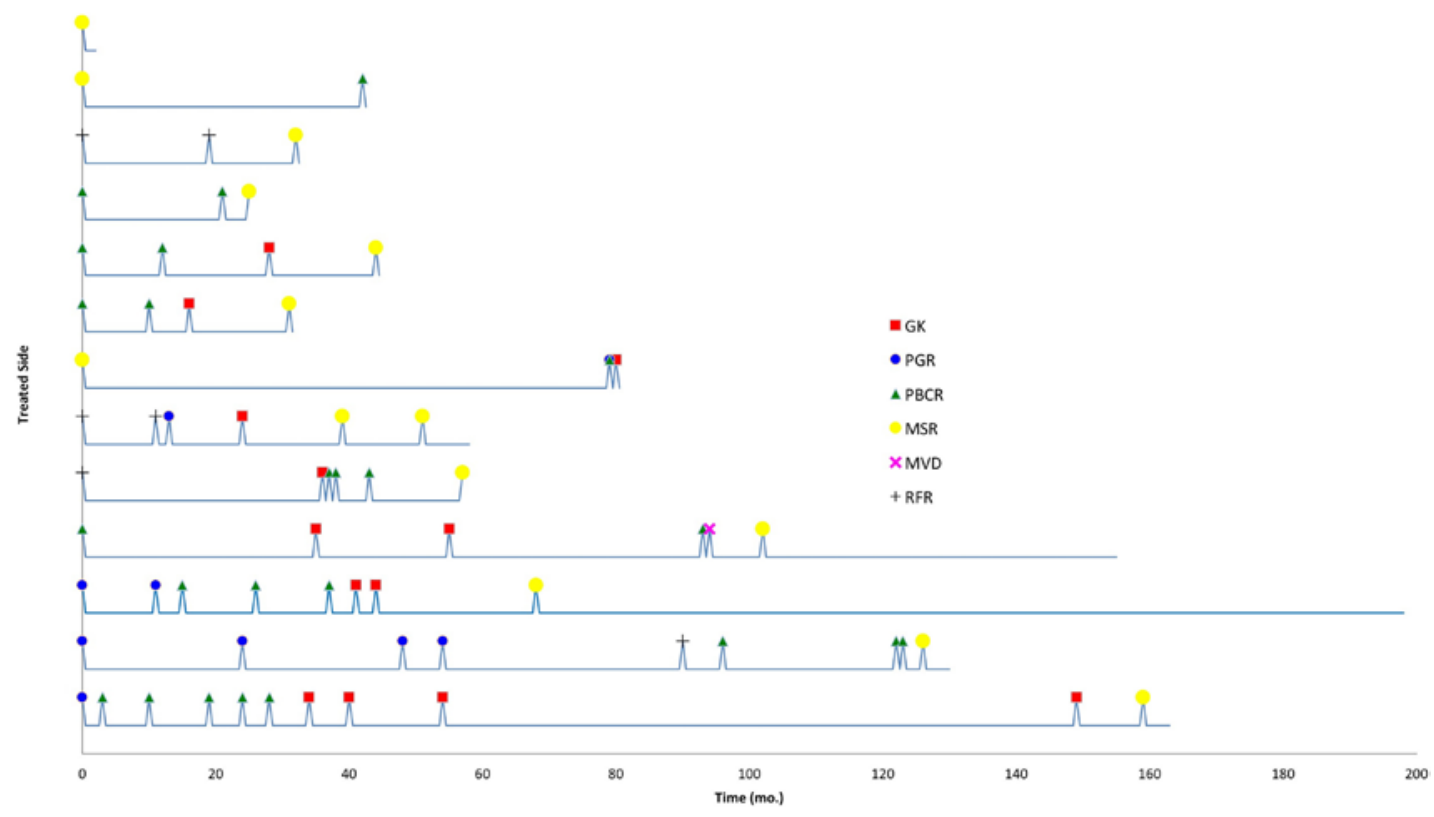

FIG. 3. Horizontal line graph illustrating time to treatment failure (TTF) in months (X-axis) for various procedures per side treated (y-axis). Circles indicate 3 patients requiring retreatment after MSR, with corresponding BNI pain scores at last follow-up. GK = Gamma Knife; MSR = microsurgical rhizotomy; MVD = microvascular decompression; PBCR = percutaneous balloon compression rhizotomy; PGR = percutaneous glycerol rhizotomy; RFR = radiofrequency rhizotomy. Figure is available in color online only.

surgery to others, are glad they underwent the procedure, felt they were better off after surgery compared to prior procedures, and wished they had undergone MSR earlier in the disease course.
Patient satisfaction scores were available for 9 of 12 patients as data for the satisfaction score were collected routinely over the past several years; 4 of 9 patients were completely satisfied with the procedure, 4 were somewhat

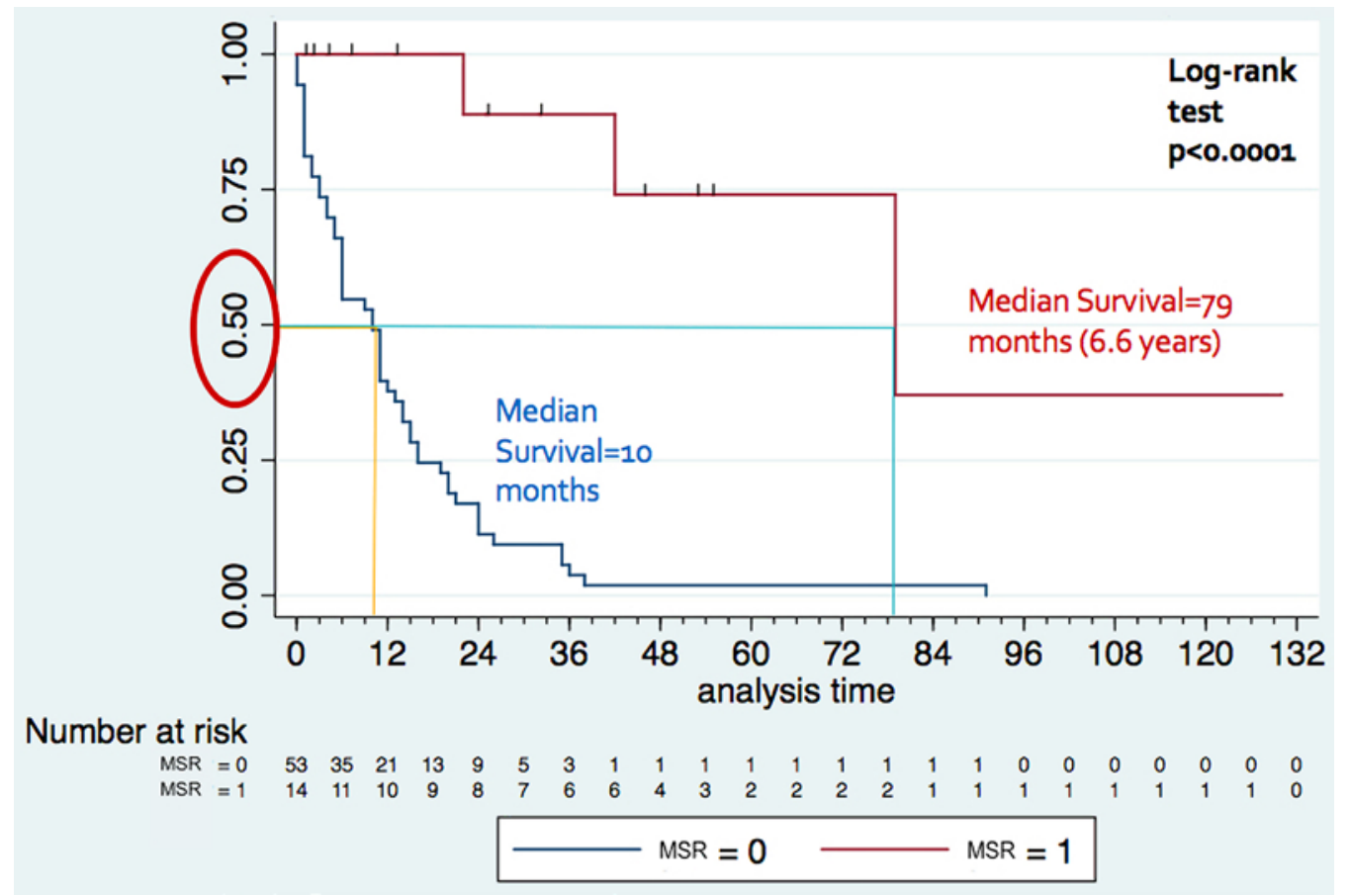

FIG. 4. Kaplan-Meier curve: TTF for MSR versus prior treatment. Survival refers to persistent benefit from treatment and thus lack of necessity for retreatment and was calculated as time to retreatment. Figure is available in color online only. 


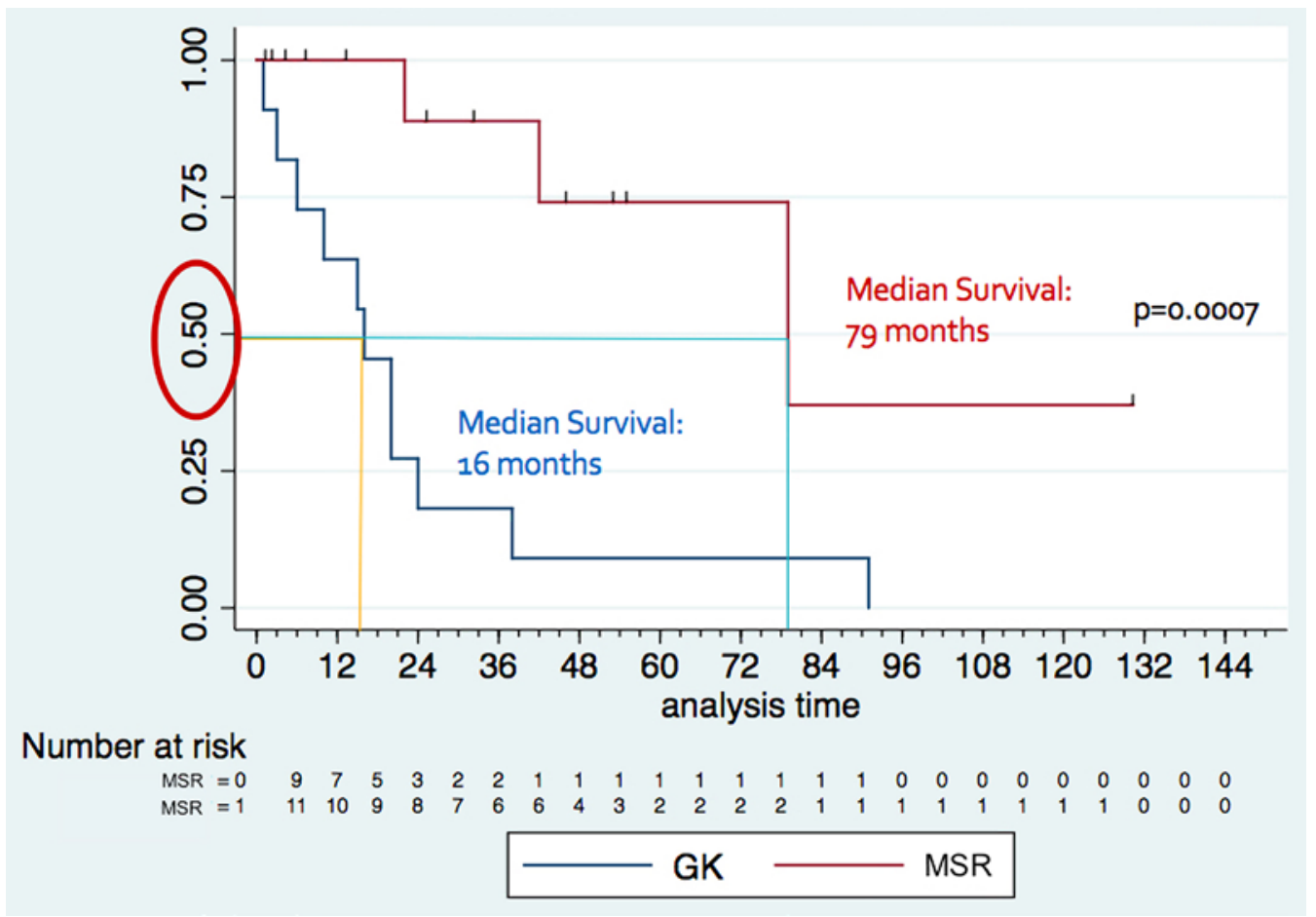

FIG. 5. Kaplan-Meier curve: TTF for MSR versus GKS in MSR cohort. Survival refers to persistent benefit from treatment and thus lack of necessity for retreatment and was calculated as time to retreatment. Figure is available in color online only.

satisfied, and 1 was completely dissatisfied. The patients' rationale for scores is described in Table 6.

\section{Discussion}

A number of treatment options exist for the manage- ment of TN, including medical management, stereotactic radiosurgery, and various percutaneous procedures, as well as the surgical options of MVD and, less commonly, MSR. The above treatment modalities, however, have been reportedly less effective in patients afflicted with MS-as-
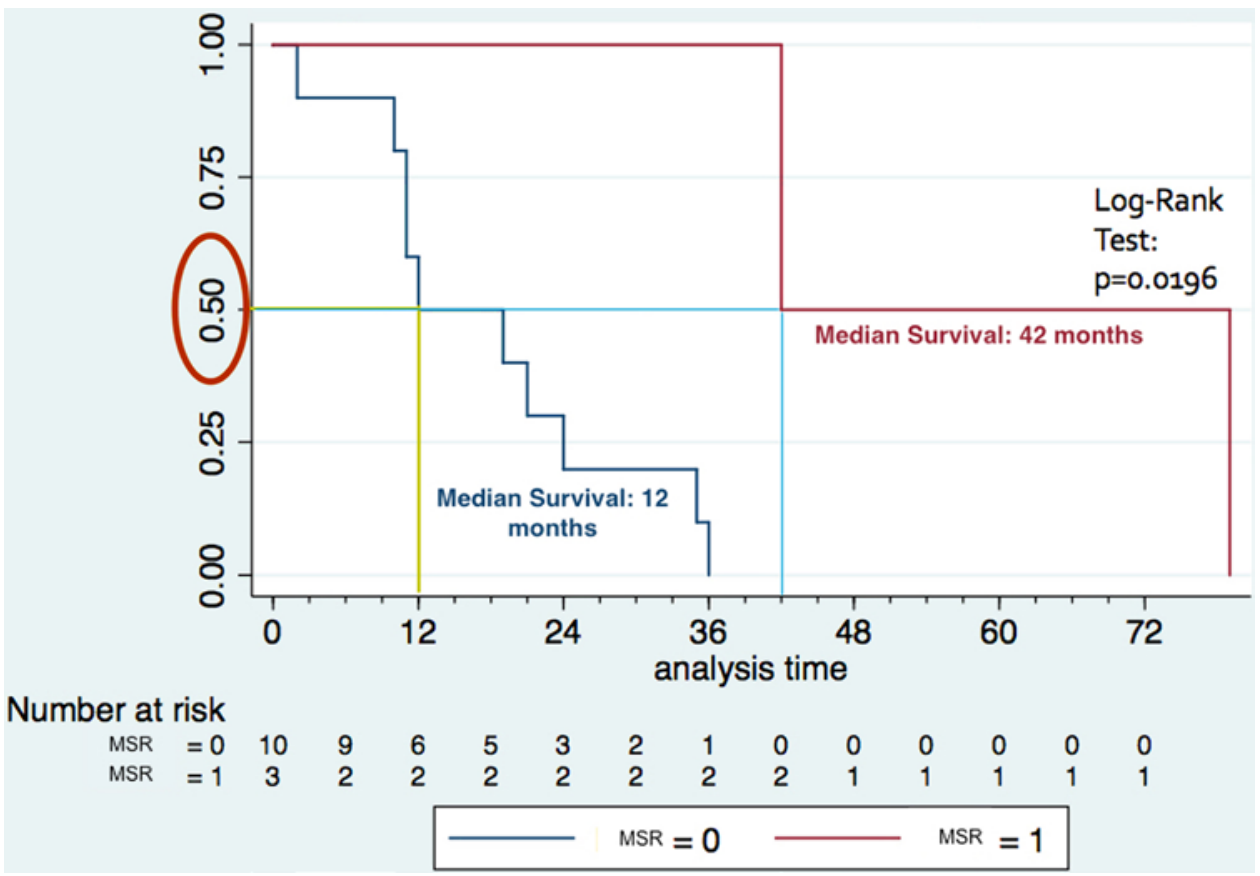

FIG. 6. Kaplan-Meier curve: TTF for MSR versus percutaneous rhizotomy as first procedure. Figure is available in color online only. 


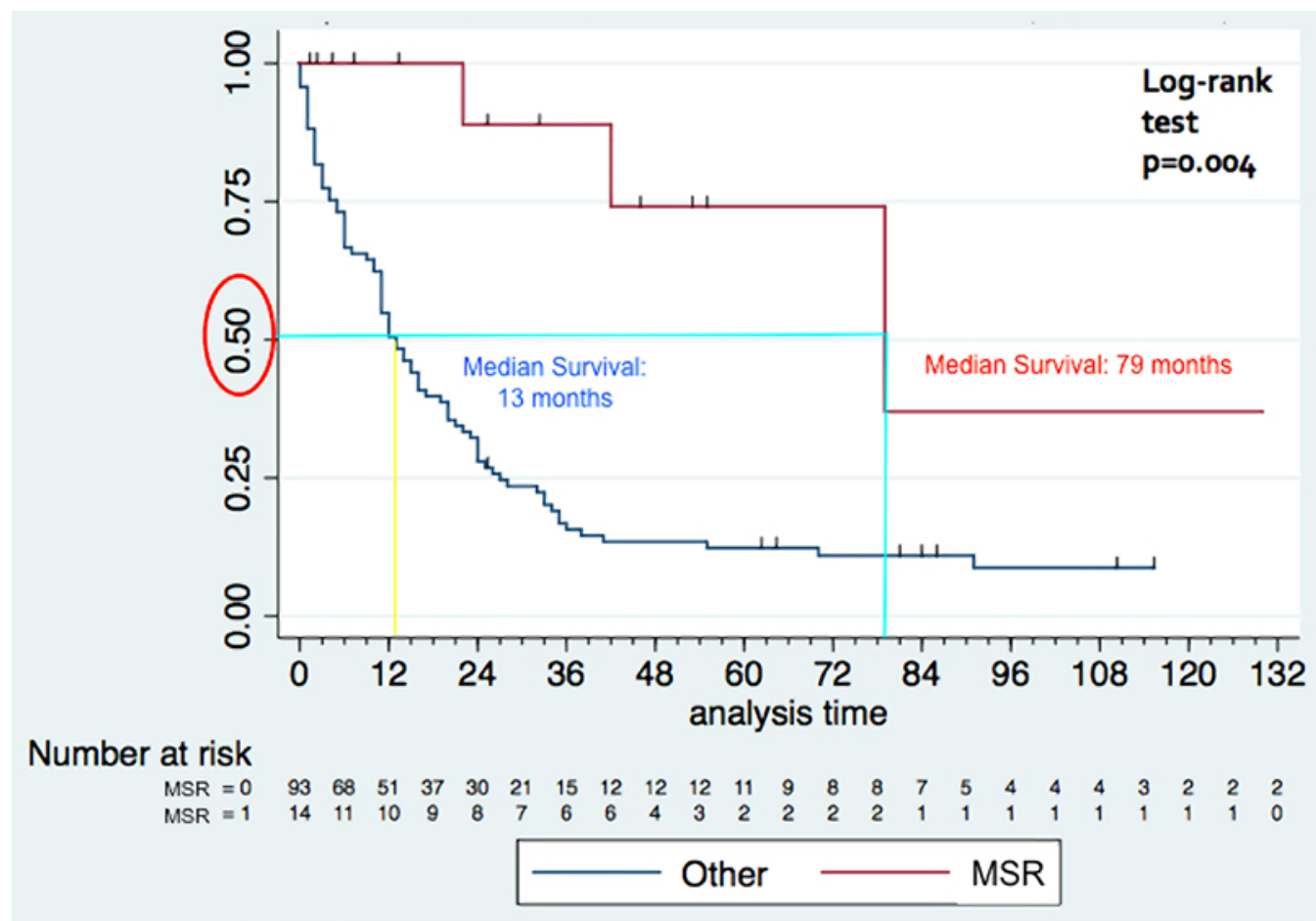

FIG. 7. Kaplan-Meier curve: TTF after MSR versus all prior procedures performed among non-MSR cohort. This cohort of MS-TN patients received various procedures but did not undergo MSR. Survival refers to persistent benefit from treatment and thus lack of necessity for retreatment and was calculated as time to retreatment. Figure is available in color online only.

sociated TN (MS-TN). Optimal treatment for this group of patients remains to be determined. Given the high rates of treatment failure, shorter pain-free intervals between treatments, and high volume of lifetime procedures, management of these patients must be carefully considered.

\section{Comparison to Literature}

The few studies that have reported results of treatment among patients afflicted with MS-TN report initial pain relief among 67\%-100\% for percutaneous techniques with recurrence and retreatment among 39\%-86\% and 33\%$70 \%$ of patients, respectively. ${ }^{14,17-20}$

Among studies of MS-TN patients undergoing GKS, initial pain relief occurred among 50\%-97\%, with pain recurrence in $39 \%-67 \%$ of patients and retreatment required in $33 \%-51 \%$ of patients in studies that reported retreatment rates. ${ }^{18,19,22-24}$

One particular study comparing percutaneous balloon compression to Gamma Knife rhizotomy reported immediate pain relief in $87 \%$ of patients undergoing balloon compression versus $23 \%$ of those treated with Gamma Knife; approximately $50 \%$ of these patients had pain relief in the early postoperative period, whereas the remaining 50\% had pain relief at 6 weeks. ${ }^{2}$ Among the patients treated with GKS who experienced postoperative pain relief, the majority (67\%) did not experience pain relief until

TABLE 6. Patient satisfaction scores

\begin{tabular}{|c|c|c|c|c|c|}
\hline Question & 5: Completely Satisfied & 4: Somewhat Satisfied & $\begin{array}{c}\text { 3: Neither Satisfied } \\
\text { nor Dissatisfied }\end{array}$ & $\begin{array}{l}\text { 2: Somewhat } \\
\text { Dissatisfied }\end{array}$ & $\begin{array}{l}\text { 1: Completely } \\
\text { Dissatisfied }\end{array}$ \\
\hline $\begin{array}{l}\text { Overall satisfaction } \\
\quad \text { with the surgery? } \\
\text { (no. of responders) }\end{array}$ & 4 & 4 & 0 & 0 & 1 \\
\hline $\begin{array}{l}\text { Primary reason for } \\
\text { satisfaction rating? }\end{array}$ & $\begin{array}{l}\text { "Pain free," "Pain relief," } \\
\text { "Pain is gone, proce- } \\
\text { dure went well, happy } \\
\text { with care and met } \\
\text { expectations" }\end{array}$ & $\begin{array}{l}\text { "Occasional abnormal facial sensations } \\
\text { make me fearful that pain may come } \\
\text { back," "Tingling feeling in jaw sometimes } \\
\text { gives me anxiety that pain will return," } \\
\text { "Was completely satisfied until pain } \\
\text { returned" (after } 4.5 \text { yrs) }\end{array}$ & NA & NA & $\begin{array}{c}\text { Pain recurrence } \\
\text { at } 42 \text { mos }\end{array}$ \\
\hline
\end{tabular}

Patient satisfaction surveys were available for 9 of 12 patients. Patients were asked about their overall satisfaction with the procedure and primary reason for the selected score. 
2-6 months following the procedure. The median recurrence time among patients who experienced initial pain relief was 10 months for the percutaneous balloon compression group compared to 18 months in the GKS group. This study is congruent with other studies in the literature which demonstrate a delayed treatment effect with GKS compared to percutaneous techniques.

Among MS-TN patients undergoing MSR, initial pain relief, pain recurrence, and retreatment were reported among $87 \%-100 \%, 15 \%-37 \%$, and $13 \%$, respectively. ${ }^{1,3}$ Where retreatment was reported, 1 of 8 patients undergoing MSR required retreatment. It is important to note that retreatment and recurrence rates are typically higher in studies with longer follow-up; therefore, the above efficacy and recurrence rates are difficult to interpret and compare given the variable follow-up duration along with differences in study size and outcome measures, inhomogeneous patient population, indications for retreatment, and methods of pain assessment.

In this study, we compare time to treatment failure (TTF) for MSR to TTF for previous procedures in the same patient cohort for ease of comparison, along with probability of treatment failure at various time points. We subsequently compare results of MSR to a second cohort of MS-TN patients undergoing multimodality treatment but not MSR.

\section{Strengths and Limitations}

The Winnipeg Centre for Cranial Nerve Disorders serves as the sole referral center for MS-TN patients for the province of Manitoba, which has among the highest prevalence rates (261 per 100,000) of MS in the world. ${ }^{16}$ Additionally, we receive multiple referrals annually from across the country. All procedures for TN in the province are performed by a single surgeon (A.M.K.) and the patients are followed clinically long-term. The above factors provide the opportunity to analyze a relatively high case volume of procedures in the MS-TN population which represents all such procedures performed in the province of Manitoba. We provide reasonably complete follow-up data on a number of surgical outcomes as well as longterm follow-up data for multiple outcome measures over the course of the patient's disease, including BNI pain scores, complications, TTF, and results of a patient satisfaction survey.

Among the limitations of this study is the relatively small sample size of 12 patients (13 sides, 14 procedures) and resulting low statistical power. Despite the small sample size, however, we observed a statistically significant difference in TTF and proportion of patients with excellent outcomes between MSR and previous procedures. The retrospective nature of this study and inherent biases of this study type limit the strength of the conclusions that can be drawn from our data.

A common clinical outcome measure, the Barrow Neurological Institute pain intensity score, was recorded for all patients postoperatively and at last follow-up. These data were not available for all patients or for all follow-up intervals after each prior procedure and therefore were not used in the analysis of prior procedures over time in the MSR cohort. We found the BNI pain score to be a dif- ficult outcome measure to analyze over time in this group given the longitudinal nature of our study, with multiple retreatments, varying pain scores throughout the disease course, varying follow-up duration and intervals, and the fact that a number of patients underwent prior procedures at other institutions across the country. We elected to analyze TTF as our primary outcome measure as we felt that it provided the most objective measure of disease severity, persistence, and impact on quality of life in this patient population. Patients, along with the treating team, elected to proceed with retreatment when it was felt that pain was sufficiently severe and persistent to accept the risks of a repeat procedure and warrant additional intervention. Retreatment was routinely offered to patients with BNI scores of 4 or 5 and in cases in which patients were unable to tolerate side effects of increased medication doses required for adequate pain control; the majority of retreatments were for pain with a BNI pain intensity score of 5 . We acknowledge that our outcome measure of TTF does not allow us to quantify the percentage of patients with complete pain relief, duration of pain-free intervals, or time to pain recurrence for previous procedures in the MSR cohort, which are commonly reported outcomes in the literature. As demonstrated by our long-term results, the disease course in the MS-TN population is characterized by years of high-dose medication use, inadequately controlled pain, and, commonly, multiple minimally effective retreatments with various procedures. We feel that TTF as an outcome measure provides the most objective means to assess procedural efficacy and durability in this context. We did, however, supplement our TTF analysis with a patient satisfaction survey, analysis of BNI pain scores, and comparison of results to a second cohort of MS-TN patients, which support our primary analysis; we observed a statistically significant difference in the proportion of patients achieving an excellent pain outcome (BNI pain score of 1 or 2) after MSR versus other prior procedures, as well as positive patient satisfaction scores. The patient satisfaction survey was administered with the aim of capturing patients' perceptions of outcomes and overall level of satisfaction with the procedure, in addition to the objective measures described above. Patient satisfaction surveys and telephone follow-up were administered by a neurosurgery clinic nurse not involved in the study in an effort to minimize reporting bias by patients and investigators.

One of the assumptions made in the analysis of the data is that each procedure is an independent event that did not impact future results of additional procedures or MSR in cases in which it was used after several prior procedures. We addressed this by referencing a number of studies that demonstrate reduced efficacy with repeat procedures. We then compared first percutaneous procedures versus last percutaneous procedures and found that TTF was significantly shorter after subsequent procedures than after a first procedure $(\mathrm{p}=0.01)$. This would suggest that any bias introduced by our assumption would be toward the null hypothesis, which would minimize the differences observed between MSR and other procedures. Additionally, we compared cases in which MSR was performed as the first procedure and compared the results to those of other first treatments, which again demonstrated a longer time to treatment failure among patients receiving MSR $(\mathrm{p}=0.02)$. 
The aim of this study was to determine whether MSR could result in improved pain outcomes and longer time to retreatment compared to prior treatment strategies employed to control the patient's pain. We subsequently analyzed GKS versus MSR and percutaneous techniques versus MSR to ensure that we were not making any faulty assumptions. We achieved statistically significant results in these subgroup analyses, with the end result being an increased TTF after MSR when compared to all prior procedures, previous percutaneous procedures, and previous Gamma Knife procedures. Our decision to combine the various treatment modalities is supported by a number of studies that suggest similar treatment efficacy between the various percutaneous techniques; 5,14 we did come across at least 1 study, however, that suggested that GKS may provide superior long-term relief compared to percutaneous balloon compression. ${ }^{2}$ For this reason, we performed a separate analysis comparing GKS versus MSR and again demonstrated a significantly longer TTF.

There are a number of limitations in our ability to comment on complications following MSR versus prior procedures. First, there were a number of patients who were referred to our center from other provinces in which they underwent prior procedures before subsequent referral. As a result, there is a possibility that we did not have access to complication data pertaining to prior procedures. Consequently, we may be understating the complication risk associated with each prior percutaneous procedure the patient may have undergone. Furthermore, the assumption regarding independence of treatment effects with multiple procedures is more likely to be a limitation when comparing complication rates between MSR and prior procedures. If a patient developed a complication after a percutaneous procedure, the patient or clinician would then be unlikely to be able to attribute the same complication to a subsequent procedure, as in the case of corneal abrasion or dysesthesia. This may result in the underestimation of complications after subsequent procedures, including MSR. We can conclude that we did not have any major complications arising from MSR; however, our ability to make comparisons with regard to complication rates between procedures is limited.

Lastly, we were able to achieve excellent pain outcomes after MSR in the majority of patients with increased TTF compared to prior procedures. We have a relatively high case volume of MVD procedures in general compared to other centers across the country and believe that case volume is an important factor in patient outcomes. Accordingly, it is uncertain whether our results can be generalized to lower-volume centers. We recommend that patients with refractory TN be treated at centers with a high case volume and extensive surgeon experience.

\section{Future Direction}

This analysis of our own cases and review of the literature suggests the need for ongoing studies with larger patient populations to determine the most durable, efficacious, cost-effective, and safest treatment strategy for managing refractory $\mathrm{TN}$ in the MS population.

\section{Conclusions}

This study provides long-term follow-up throughout the disease course for a cohort of patients undergoing MSR, typically after multiple failed multimodality treatments, for refractory MS-associated TN. Time to treatment failure was significantly prolonged in patients undergoing MSR compared to percutaneous procedures and Gamma Knife. Median survival of treatment was considerably longer after MSR versus other procedures. A significantly higher proportion of patients were pain-free after MSR versus prior procedures. Probability of treatment survival was significantly higher at all time points after MSR. There were no major complications after MSR, and there did not appear to be an increased risk of bothersome dysesthesia, anesthesia dolorosa, or corneal erosion. Based on our experience, MSR provides superior pain outcomes and longer time to retreatment compared to Gamma Knife and percutaneous procedures and should be considered earlier in the disease course for patients with refractory MS-associated TN. In recent years, we have begun to offer MSR earlier in the disease course for patients with refractory MS-TN at our institution rather than as a "last-ditch" effort after years of failed procedures, as we had done in past years. In the small group of patients with recurrence after initial MSR, repeat MSR appears to be more effective than repeat percutaneous or Gamma Knife procedures which failed to provide pain relief.

\section{Acknowledgments} sketch.

We thank Jon Stepaniuk for providing his original illustrative

\section{References}

1. Abhinav K, Love S, Kalantzis G, Coakham HB, Patel NK: Clinicopathological review of patients with and without multiple sclerosis treated by partial sensory rhizotomy for medically refractory trigeminal neuralgia: a 12-year retrospective study. Clin Neurol Neurosurg 114:361-365, 2012

2. Alvarez-Pinzon AM, Wolf AL, Swedberg HN, Barkley KA, Cucalon J, Curia L, et al: Comparison of percutaneous retrogasserian balloon compression and Gamma Knife radiosurgery for the treatment of trigeminal neuralgia in multiple sclerosis. World Neurosurg 97:590-594, 2017

3. Antic B, Peric P: Posterior fossa exploration in treatment of trigeminal neuralgia associated with multiple sclerosis. Surg Neurol 71:419-423, 2009

4. Attabib N, Kaufmann AM: Use of fenestrated aneurysm clips in microvascular decompression surgery. Technical note and case series. J Neurosurg 106:929-931, 2007

5. Bender MT, Pradilla G, Batra S, See AP, James C, Pardo CA, et al: Glycerol rhizotomy and radiofrequency thermocoagulation for trigeminal neuralgia in multiple sclerosis. J Neurosurg 118:329-336, 2013

6. Bigder MG, Kaufmann AM: Failed microvascular decompression surgery for hemifacial spasm due to persistent neurovascular compression: an analysis of reoperations. J Neurosurg 124:90-95, 2016

7. Brisman R: Trigeminal neuralgia and multiple sclerosis. Arch Neurol 44:379-381, 1987

8. Campos-Benitez M, Kaufmann AM: Neurovascular compression findings in hemifacial spasm. J Neurosurg 109:416-420, 2008

9. Conti A, Pontoriero A, Iatì G, Esposito F, Siniscalchi EN, 
Crimi S, et al: Frameless stereotactic radiosurgery for treatment of multiple sclerosis-related trigeminal neuralgia.

World Neurosurg 103:702-712, 2017

10. Eldridge PR, Sinha AK, Javadpour M, Littlechild P, Varma TR: Microvascular decompression for trigeminal neuralgia in patients with multiple sclerosis. Stereotact Funct Neurosurg 81:57-64, 2003

11. Headache Classification Committee of the International Headache Society (IHS): The International Classification of Headache Disorders, 3rd edition (beta version). Cephalalgia Int J Headache 33:629-808, 2013

12. Jensen TS, Rasmussen P, Reske-Nielsen E: Association of trigeminal neuralgia with multiple sclerosis: clinical and pathological features. Acta Neurol Scand 65:182-189, 1982

13. Kaufmann AM, Wilkinson MF: Microvascular decompression surgery for hemifacial spasm, in Schmidek HH (ed): Schmidek \& Sweet Operative Neurosurgical Techniques: Indications, Methods, and Results, ed 5. Philadelphia: Elsevier, 2005

14. Mallory GW, Atkinson JL, Stien KJ, Keegan BM, Pollock BE: Outcomes after percutaneous surgery for patients with multiple sclerosis-related trigeminal neuralgia. Neurosurgery 71:581-586, 2012

15. Manzoni GC, Torelli P: Epidemiology of typical and atypical craniofacial neuralgias. Neurol Sci 26 (Suppl 2):s65-s67, 2005

16. Marrie RA, Yu N, Blanchard J, Leung S, Elliott L: The rising prevalence and changing age distribution of multiple sclerosis in Manitoba. Neurology 74:465-471, 2010

17. Martin S, Teo M, Suttner N: The effectiveness of percutaneous balloon compression in the treatment of trigeminal neuralgia in patients with multiple sclerosis. J Neurosurg 123:1507-1511, 2015

18. Mathieu D, Effendi K, Blanchard J, Séguin M: Comparative study of Gamma Knife surgery and percutaneous retrogasserian glycerol rhizotomy for trigeminal neuralgia in patients with multiple sclerosis. J Neurosurg 117 Suppl:175-180, 2012

19. Mohammad-Mohammadi A, Recinos PF, Lee JH, Elson P, Barnett GH: Surgical outcomes of trigeminal neuralgia in patients with multiple sclerosis. Neurosurgery 73:941-950, 2013

20. Montano N, Papacci F, Cioni B, Di Bonaventura R, Meglio $\mathrm{M}$ : Percutaneous balloon compression for the treatment of trigeminal neuralgia in patients with multiple sclerosis. Analysis of the potentially prognostic factors. Acta Neurochir (Wien) 154:779-783, 2012

21. Moulin DE, Foley KM, Ebers GC: Pain syndromes in multiple sclerosis. Neurology 38:1830-1834, 1988

22. Rogers CL, Shetter AG, Ponce FA, Fiedler JA, Smith KA, Speiser BL: Gamma knife radiosurgery for trigeminal neuralgia associated with multiple sclerosis. J Neurosurg 97 (5 Suppl):529-532, 2002

23. Tuleasca C, Carron R, Resseguier N, Donnet A, Roussel P, Gaudart J, et al: Multiple sclerosis-related trigeminal neuralgia: a prospective series of 43 patients treated with Gamma Knife surgery with more than one year of follow-up. Stereotact Funct Neurosurg 92:203-210, 2014

24. Zorro O, Lobato-Polo J, Kano H, Flickinger JC, Lunsford LD, Kondziolka D: Gamma knife radiosurgery for multiple sclerosis-related trigeminal neuralgia. Neurology 73:11491154,2009

\section{Disclosures}

The authors report no conflict of interest concerning the materials or methods used in this study or the findings specified in this paper.

\section{Author Contributions}

Conception and design: Bigder, Kaufmann. Acquisition of data: Bigder, Krishnan. Analysis and interpretation of data: Bigder. Drafting the article: Bigder. Critically revising the article: Bigder, Cook, Kaufmann. Reviewed submitted version of manuscript: Bigder, Krishnan, Kaufmann. Approved the final version of the manuscript on behalf of all authors: Bigder. Statistical analysis: Bigder. Study supervision: Cook, Kaufmann.

\section{Supplemental Information Videos}

Video 1. https://vimeo.com/253259824.

\section{Correspondence}

Mark G. Bigder: University of Manitoba, Winnipeg, MN, Canada. umbigder@myumanitoba.ca. 